\title{
Association of tryptophan hydroxylase-2 polymorphisms with oppositional defiant disorder in a Chinese Han population
}

\author{
Chang-Hong Wang ${ }^{1 \dagger}$, Cong Liu ${ }^{1 \dagger}$, En-Zhao Cong ${ }^{1}$, Gai-Ling Xu' ${ }^{1}$ Ting-Ting Lv' ${ }^{1}$, Ying-Li Zhang ${ }^{1}$, Qiu-Fen Ning ${ }^{1}$, \\ Ji-Kang Wang ${ }^{1}$, Hui-Yao Nie ${ }^{1 * \ddagger}$ and Yan $\mathrm{Li}^{2^{* \neq}}$
}

\begin{abstract}
Background: Oppositional defiant disorder (ODD) is a behavioral disorder of school-age population. It is well known that 5-HT dysfunction is correlated with impulsivity, which is one of the common characteristics of ODD. The enzyme tryptophan hydroxylase-2 (TPH-2) synthesizes 5-HT in serotonergic neurons of the midbrain raphe. The purposes of this study were to investigate the potential association of TPH-2 polymorphisms with susceptibility to ODD in a Han Chinese school population.

Methods: Four polymorphisms (rs4570625, rs11178997, rs1386494 and rs7305115) of the TPH-2 gene were analyzed by using polymerase chain reaction and DNA microarray hybridization in a case-control study of 276 Han Chinese individuals (124 ODD and 152 controls).

Results: In single marker analyses, there was a significant difference in the genotype $\left(x^{2}=4.163, P=0.041\right)$ and allele frequency $\left(X^{2}=3.930, P=0.047\right)$ of rs 1386494 between ODD and control groups. Haplotype analyses revealed higher frequencies of haplotypes TA (rs4570625-rs11178997), TAG (rs4570625-rs11178997-rs1386494), TAA (rs4570625-rs11178997-rs7305115) and TAGA (rs4570625-rs11178997-rs1386494-rs7305115), but lower frequencies of haplotypes GA (rs4570625-rs11178997) and GAG (rs4570625-rs11178997-rs1386494) in ODD compared to control groups.
\end{abstract}

Conclusions: These findings suggest the role of these TPH-2 gene variants in susceptibility to ODD. Some haplotypes might be the risk factors for Chinese Han children with ODD, while others might be preventable factors.

Keywords: Oppositional defiant disorder, Tryptophan hydroxylase-2 gene, Single nucleotide polymorphisms

\section{Background}

Oppositional defiant disorder (ODD) is a behavioral disorder mainly characterized by resistance, disobedience, provocation or hostility to authority figures during growth and development in children and adolescents [1, 2]. Children and adolescents with ODD may have trouble

\footnotetext{
*Correspondence: niehuiyao@163.com; liyanzzu2009@126.com ${ }^{+}$Chang-Hong Wang and Cong Liu contributed equally to this work ${ }^{\ddagger}$ Yan Li and Hui-Yao Nie have contributed equally to this work ${ }^{1}$ Department of Psychiatry, The Second Affiliated Hospital of Xinxiang Medical University (Psychiatric hospital of Henan province, China), Xinxiang 453002, Henan, China

${ }^{2}$ Department of Child and Adolescent, Public Health College, Zhengzhou University, 100 Kexue Road, Zhengzhou 450001, Henan, China
}

controlling their temper, showing intense emotional reaction or impulsive actions in response to mild stimulation. Thus, ODD is considered as a disorder of emotional regulation [3]. Children suffering from ODD are at risk for numerous negative outcomes, such as delinquency, unemployment, depression, anxiety and other psychiatric problems [4]. However, the pathological mechanisms of ODD are still unclear.

Based on DSM-IV-TR, the prevalence rate for ODD is $2-16 \%$ [5]. Well established in previous research, this disorder exhibits moderate heritability, and is substantially stable over time, particularly through childhood [6], and genetic underpinning is an important factor which can influence children's disruptive behavior, like ODD [7]. 
Familial clustering suggests an underlying genetic component, but hereditary connections are variable [1]. ODD has been consistently associated with attention-deficit/ hyperactivity disorder (ADHD) $[8,9]$ and conduct disorder (CD) [10-13]. The estimated heritability of ADHD is approximately 0.76 [14] and $40-60 \%$ of ADHD were also diagnosed with ODD [15], suggesting that ODD might share common genetic mechanisms with ADHD [8]. However, the comorbidity of ODD may influence the clinical characteristics, progression and treatment response for ADHD cases [14].

Serotonin (5-HT) is a neurotransmitter involved in various bodily functions, such as aggression, attention, appetite and locomotion. The deficiency of the 5 -HT functions is related to depression, anxiety, irregular appetite, aggression, increased pain sensation, and ADHD symptoms [16]. Especially, 5-HT dysfunction is correlated with impulsivity, which is one of the common characteristics of ADHD, ODD, personality disorder [17] and substance abuse [18, 19]. Early studies reported a clear association between low cerebrospinal fluid 5-HT and impulsive aggression [20]. The conversion of tryptophan to 5-hydroxytryptophan is the first and rate limiting step in 5-HT synthesis catalyzed by two subtypes of the enzyme tryptophan hydroxylase (TPH-1 and TPH-2); 5 -HT is then formed by decarboxylation of 5-hydroxytryptophan. The studies revealed differential expression of classical TPH-1 synthesizing 5-HT in peripheral tissues, and TPH-2 synthesizing 5-HT mainly in serotonergic neurons of the midbrain raphe [21]. In mice brain stems, the expression of TPH-1 appears to be 150 times lower than TPH-2 [21], suggesting that TPH-2 may play a much more important role in serotonin synthesis in the brain than TPH-1. Thus, the studies have focused on the role of brain-specific TPH-2 in the pathophysiology of various psychiatric disorders, including ADHD [16].

The human TPH-2 gene spans less than $100 \mathrm{~kb}$, consists of 11 exons and is located in the chromosome 12 q21.1 region. Several studies have explored the association between TPH-2 gene polymorphisms and ADHD. For example, Sheehan et al. [22] firstly reported the association between TPH2-rs1843809 and ADHD through a family study. A subsequent study reported association between TPH2-rs4570625 or TPH2-rs11178997 and ADHD through a family study [23]. A more recent study showed that a significant correlation between the frequencies of the rs11179027 and rs1843809 of alleles of TPH-2 and ADHD [16]. In addition, the TPH-2 gene polymorphism have been found to be associated with lateonset depression [24], PTSD [25, 26], suicide in patients with alcohol dependence [27, 28] and suicidal behavior [29], as well as with schizophrenia [30,31] and panic in bipolar disorders [32] in the Chinese Han population.
In view of the possible shared common genes between ADHD and ODD, the important role of TPH-2 in 5-HT synthesis in brain and the possible association between TPH-2 gene polymorphisms and ADHD, as well as the associations of the TPH-2 gene polymorphism with behavioral and psychiatric disorders in previous studies, it would be of interest to examine the association between TPH-2 gene polymorphisms and ODD, which, to our best knowledge, has not been reported. Therefore, the main purpose of the current study was to examine whether the TPH-2 gene polymorphisms was associated with the susceptibility to ODD in a Chinese Han population.

\section{Methods \\ Subjects}

Using the random group sampling method, 2000 Chinese Han students in primary school in Nanyang, Henan Province, China were assessed with Conners Teachers Rating Scale between 2007 and 2009, all four grandparents and both parents of each child were known to be of Han Chinese origin. To confirm the diagnosis of ODD, one or both parents and teachers were interviewed by a chief physician and resident physician on the basis of DSM-IV diagnostic criteria. Inclusion criteria were: (a) aged 6-14 years; (b) had ODD symptoms at least 6 months; (c) intelligence quotient (IQ) $\geq 70$ based on Raven's Progressive Matrices; (d) no physical diseases, mental retardation, low body mass index (BMI, $<18.5 \mathrm{~kg} /$ $\mathrm{m}^{2}$ ) or other mental illnesses, or ADHD or CD symptoms. A total of 125 children were confirmed with ODD diagnosis. Among them, 124 subjects were enrolled in the study, including 70 boys (56.5\%) and 54 girls (43.5\%) with an average age of $10.4 \pm 1.9$ years.

Data also consisted of 152 control subjects (boy/ girl $=78 / 74$ ), who were recruited from the same primary school. Mean age was $10.5 \pm 1.6$ years. Inclusion criteria were: (a) aged 6-14 years; (b) no any ODD symptoms; (c) intelligence quotient (IQ) $\geq 70$ based on Raven's Progressive Matrices; (d) no physical diseases, mental retardation, low body mass index (BMI, $<18.5 \mathrm{~kg} / \mathrm{m}^{2}$ ) or other mental illnesses, or ADHD or CD symptoms.

There was no significant differences in gender, age and education between ODD and control groups (all $P>0.05)$. This study was approved by the Ethical Committee of the Second Affiliated Hospital, Xinxiang Medical College, Henan Province. Informed consent was obtained from all subjects and their parents.

\section{$T P H-2$ genotyping}

$5 \mathrm{ml}$ blood samples were collected from cubital vein between 8:00 and 9:00 a. m. following an overnight fast and placed into the tubes with EDTA anticoagulant. Samples were stored at $-70{ }^{\circ} \mathrm{C}$ until assayed. 
DNA was extracted using a Genomic DNA extraction kit (DP318) (TIANGEN biotechnology company, Beijing, China). The DNA was amplified by polymerase chain reaction (PCR) methods and the primers for the four loci (rs4570625, rs11178997, rs1386494 and rs7305115) were designed by Invitrogen Corporation (Shanghai, China). Oligonucleotide sequences are presented in Table 1.

The total volume of the PCR reaction was $30 \mu \mathrm{l}$ which contained $0.5 \mu \mathrm{l}$ whole genome DNA $(50 \mathrm{ng} / \mu \mathrm{l}), 3 \mu \mathrm{l}$ $10 \times$ PCR buffer solution, $0.5 \mu \mathrm{l} 10 \mathrm{~mm} \mathrm{~L}^{-1} \mathrm{dNTPs}, 0.5 \mu \mathrm{l}$ each primer, $0.3 \mu \mathrm{l} \mathrm{Taq}$ polymerase, $1.5 \mu \mathrm{l} 25 \mathrm{~mm} \mathrm{~L}^{-1}$ $\mathrm{MgCl}_{2}$, and $23.7 \mu \mathrm{l}$ sterile water. Loop parameters for PCR were as follows: initial denaturation at $95{ }^{\circ} \mathrm{C}$ for $5 \mathrm{~min}$, amplification at $94{ }^{\circ} \mathrm{C}$ for $30 \mathrm{~s}$, annealing at $54 / 56{ }^{\circ} \mathrm{C}$ for $45 \mathrm{~s}$, and extension at $72{ }^{\circ} \mathrm{C}$ for $45 \mathrm{~s}$. The process was repeated 34 times, followed by extension at $72{ }^{\circ} \mathrm{C}$ for $5 \mathrm{~min}$. PCR products were placed onto glass slides disposed with acrylamide by a Pixsys 5500 microarrayer (Cartesian Products, Inc. America) [33]. The PCR products were hybridized with fluorescence-labeled probes at $37^{\circ} \mathrm{C}$ for $5-6 \mathrm{~h}$, and the glass slides were scanned with a LuxScan-10k confocal scanner (Capitalbio Corporation, Beijing, China). The genotype of each sample was detected based on the fluorescent signals [34].

\section{Statistical analysis}

Differences between genotype groups were analyzed using Chi squared for categorical variables and the Student's $t$ test or one-way analysis of variance (ANOVA) for continuous variables using the PASW Statistics 18.0 software (SPSS Inc., Chicago, IL, USA).

Deviation from the Hardy-Weinberg equilibrium (HWE) was tested separately in cases and controls using Chi square $\left(\chi^{2}\right)$ goodness-of-fit test. The difference in the allele and genotype frequencies for $T P H-2$ polymorphisms between ODD and normal controls was analyzed using the $\chi^{2}$ test. Pairwise linkage disequilibrium (LD) between four TPH-2 markers was analyzed in cases and normal controls. Haploview 4.2 was used to compute pairwise

\section{Table 1 Primer sequences of these four loci}

\begin{tabular}{ll}
\hline SNP ID & Primer sequence $\left(\mathbf{5}^{\prime}-\mathbf{3}^{\prime}\right)$ \\
\hline rs4570625 & F: 5'-GAACCCTTACCTTTCCTTTG-3' \\
& R: 5'Acry-TCCACTCTTCCAGTTATTTT-3' \\
rs11178997 & F: 5'-GTGTTCGGGAGCACAATAAT-3' \\
& R: 5' Acry-AAGCCTGCCACTGGAAGTT-3' \\
rs1386494 & F: 5'-TGTTTCTCGCAGGTTGTTGG-3' \\
& R: 5' Acry-AGCAAATGAATCACAAAGGG-3' \\
rs7305115 & F: 5'-TAGTTGGTTTTCTGTTGC-3' \\
& R: 5'Acry-CCCTTTTCTCTTTAGGTGAG-3' \\
\hline
\end{tabular}

Sequences of the four primers
LD statistics for markers, haplotype block, haplotype frequency, and haplotype association. We used a 2-4-window fashion analysis. Rare haplotypes found in less than $3 \%$ were excluded from the association analysis. A logistic regression analysis was conducted to examine the independent association of each haplotype on the categorical diagnosis of case (0: control, 1: case) after adjusting for the confounders. To control haplotype analyses for multiple testing, 10,000 permutations were performed for the most significant tests to determine the empirical significance.

The power (power defined as the chance that true differences will actually be detected) of the sample was calculated using Quanto Software [35], with known risk allele frequencies and an ODD population prevalence of $0.02-0.16$, and we examined log additive, recessive and dominant models.

\section{Results}

\section{Single locus analysis}

The genotype and allele frequencies of four SNPs located in the TPH-2 gene are summarized in Table 6. No deviation from HWE was detected in the cases or controls (all $P>0.05$; Tables 2, 3, 4, 5). Significant differences in the genotype and allele frequencies between cases and controls were observed for rs1386494 (genotype $\chi^{2}=4.163$, $P=0.041$; allele $\chi^{2}=3.930, P=0.047$ ). The frequency of the Gallele of rs1386494 was higher in patients than in controls. There was no allelic or genotypic association between the other three SNPs and ODD (all $P>0.05$, Table 6).

\section{Linkage disequilibrium (LD) analysis}

LD analyses were performed for all polymorphism pairs in both case and control subjects. All four polymorphisms were in slight to modest $\mathrm{LD}$ or without $\mathrm{LD}$ with each other in both control $\left(\mathrm{D}^{\prime}=0.12-0.92 ; \mathrm{r}^{2}=0.02-\right.$ $0.71)$ and patient groups $\left(\mathrm{D}^{\prime}=0.10-0.76 ; \mathrm{r}^{2}=0.00-0.16\right)$ (Fig. 1).

\section{Haplotype analysis}

Two-four SNP sliding window haplotype analyses were performed. Only those haplotypes with a frequency above 3\% were included in the analyses. Estimation of haplotype frequencies and comparison of haplotype frequency distributions between cases and controls were conducted using the program Haploview.

We observed significant differences in the frequencies of TA $(P=0.014, O R=1.951,95 \% C I 1.140-3.341)$ and GA $(P=0.012, O R=0.149,95 \% C I 0.027-0.826)$ haplotypes containing rs4570625-rs11178997 between case and control groups.

Also, we noted significant differences in the frequencies of TAG $(P=0.02, O R=1.896,95 \%$ CI 1.099-3.272) 
Table 2 rs4570625 Hardy-Weinberg Equilibrium test between ODD and control group

\begin{tabular}{|c|c|c|c|c|c|c|}
\hline \multirow[t]{2}{*}{ Group } & \multicolumn{6}{|c|}{ Genotype } \\
\hline & GG & GT & $\mathrm{TT}$ & Total & $x^{2}$ & $P$ \\
\hline ODD group & & & & & 1.474 & 0.225 \\
\hline Observation (O) & 27 & 51 & 38 & 116 & & \\
\hline Expectation (E) & 23.761 & 57.478 & 34.761 & 116 & & \\
\hline Control group & & & & & 1.301 & 0.254 \\
\hline Observation (O) & 36 & 83 & 33 & 152 & & \\
\hline Expectation (E) & 39.515 & 75.970 & 36.51 & 152 & & \\
\hline
\end{tabular}

$\mathrm{df}=1$

Table 3 rs11178997 Hardy-Weinberg equilibrium test between ODD and control group

\begin{tabular}{|c|c|c|c|c|c|c|c|}
\hline \multirow[t]{2}{*}{ Group } & \multicolumn{7}{|c|}{ Genotype } \\
\hline & AA & AT & $\mathrm{TT}$ & Total & $x^{2}$ & $\mathrm{x}^{2^{*}}$ & $P$ \\
\hline ODD group & & & & & & 1.292 & 0.256 \\
\hline Observation $(\mathrm{O})$ & 6 & 33 & 84 & 123 & & & \\
\hline Expectation (E) & $4.116^{*}$ & 36.768 & 82.116 & 123 & & & \\
\hline Control group & & & & & & 0.052 & 0.820 \\
\hline Observation (O) & 4 & 39 & 109 & 152 & & & \\
\hline Expectation (E) & $3.633^{*}$ & 39.734 & 108.63 & 152 & & & \\
\hline
\end{tabular}

$\mathrm{df}=1$, when $\mathrm{E}$ is little than 5 , we use Yates corrected Chi squared test, $\mathrm{x}^{2^{*}}$

Table 4 rs 1386494 Hardy-Weinberg equilibrium test between ODD and control group

\begin{tabular}{|c|c|c|c|c|c|c|c|}
\hline \multirow[t]{2}{*}{ Group } & \multicolumn{7}{|c|}{ Genotype } \\
\hline & AA & AG & GG & Total & $x^{2}$ & $\mathrm{x}^{2^{*}}$ & $P$ \\
\hline ODD group & & & & & & 0.138 & 0.711 \\
\hline Observation (O) & 0 & 8 & 116 & 124 & & & \\
\hline Expectation (E) & $0.129 *$ & 7.742 & 116.129 & 124 & & & \\
\hline Control group & & & & & & 0.856 & 0.355 \\
\hline Observation (O) & 0 & 21 & 128 & 149 & & & \\
\hline Expectation (E) & $0.740^{*}$ & 19.520 & 128.740 & 149 & & & \\
\hline
\end{tabular}

$\mathrm{df}=1^{*}$ when $\mathrm{E}$ is little than 5 , we use Yates corrected Chi squared test, $\mathrm{x}^{2^{*}}$

Table 5 rs7305115 Hardy-Weinberg equilibrium test between ODD and control group

\begin{tabular}{|c|c|c|c|c|c|c|}
\hline Group & $A A$ & AG & GG & Total & $x^{2}$ & $P$ \\
\hline ODD group & & & & & 3.389 & 0.533 \\
\hline Observation (O) & 34 & 56 & 29 & 119 & & \\
\hline Expectation (E) & 32.303 & 59.395 & 27.302 & 119 & & \\
\hline Control group & & & & & 0.844 & 0.358 \\
\hline Observation $(\mathrm{O})$ & 35 & 64 & 40 & 139 & & \\
\hline Expectation (E) & 2.295 & 69.410 & 37.295 & 139 & & \\
\hline
\end{tabular}

$\mathrm{df}=1$ 
Table 6 Genotype and allele frequency distribution of the four loci of THP-2 between two groups

\begin{tabular}{|c|c|c|c|c|c|c|c|c|c|}
\hline \multirow{2}{*}{$\begin{array}{l}\text { Locus } \\
\text { rs4570625 }\end{array}$} & \multicolumn{3}{|c|}{ Genotype (\%) } & \multirow{2}{*}{$\begin{array}{l}x^{2} \\
4.525\end{array}$} & \multirow{2}{*}{$\begin{array}{l}P \\
0.104\end{array}$} & \multicolumn{2}{|l|}{ Allele (\%) } & \multirow{2}{*}{$\frac{x^{2}}{1.729}$} & \multirow{2}{*}{$\frac{P}{0.189}$} \\
\hline & GG & GT & $\mathrm{TT}$ & & & G & $\mathrm{T}$ & & \\
\hline ODD & $27(23.3)$ & $51(44.0)$ & $38(32.8)$ & & & $105(45.3)$ & $127(54.7)$ & & \\
\hline Control & $36(23.7)$ & $83(54.6)$ & $33(21.7)$ & & & $155(51.0)$ & $149(49.0)$ & & \\
\hline rs11178997 & $\mathrm{AA}$ & AT & $\mathrm{TT}$ & 1.092 & 0.579 & A & $\mathrm{T}$ & 0.783 & 0.376 \\
\hline ODD & $6(4.9)$ & $33(26.8)$ & $84(68.3)$ & & & $45(18.3)$ & $201(81.7)$ & & \\
\hline Control & $4(2.6)$ & $39(25.7)$ & $109(71.7)$ & & & $47(15.5)$ & $257(84.5)$ & & \\
\hline rs1386494 & $\mathrm{AA}$ & $A G$ & GG & 4.163 & $0.041^{*}$ & A & G & 3.930 & $0.047^{*}$ \\
\hline ODD & 0 & $8(6.5)$ & $116(93.5)$ & & & $8(3.2)$ & $240(96.8)$ & & \\
\hline Control & 0 & $21(14.1)$ & $128(85.9)$ & & & $21(7.0)$ & $277(93.0)$ & & \\
\hline rs7305115 & $A A$ & $A G$ & GG & 0.756 & 0.685 & A & G & 0.780 & 0.377 \\
\hline ODD & 34 (28.6) & $56(47.1)$ & $29(24.4)$ & & & $124(52.1)$ & $114(47.9)$ & & \\
\hline Control & $35(25.2)$ & $64(46.0)$ & $40(28.8)$ & & & $134(48.2)$ & $144(51.8)$ & & \\
\hline
\end{tabular}

* $P<0.05$
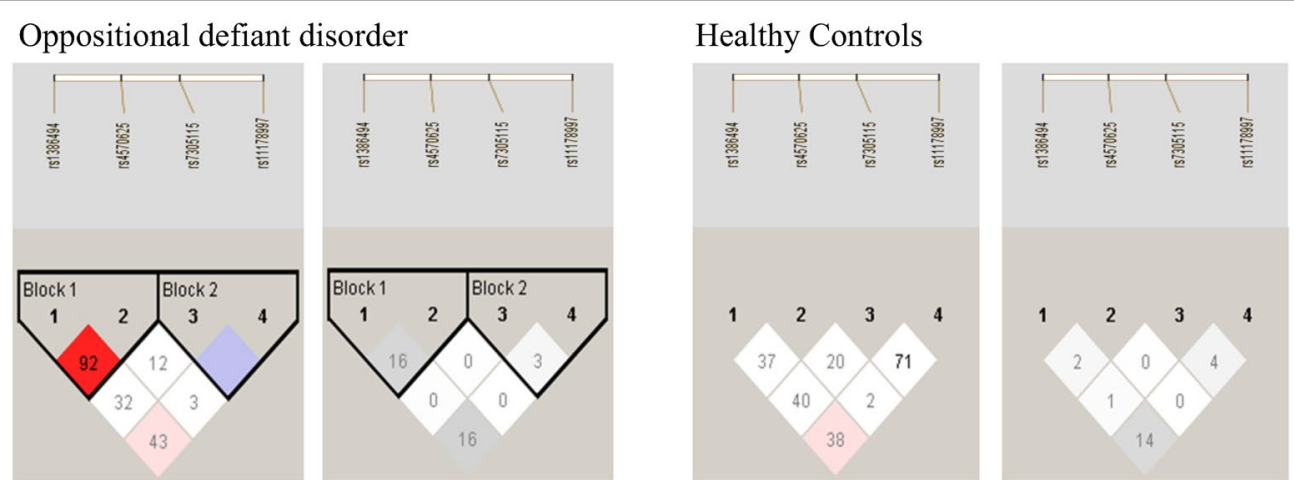

Fig. 1 Genomic structure of TPH-2, including relative location of 4 SNPs studied and linkage disequilibrium (LD) of these four SNPs in the oppositional defiant disorder (ODD) and control groups. The LD between pairwise SNPs, using D' (left, red color) and r ${ }^{2}$ (right, gray color) values, are shown separately for cases and controls. High levels of LD are represented by increasing scale intensity from 0 to 100 , as shown by the bars

and GAG $(P=0.013, O R=0.149,95 \%$ CI $0.027-0.831)$ containing rs4570625-rs11178997-rs1386494, as well as TAA $(P=0.026, O R=2.315,95 \%$ CI $1.088-4.927)$ containing rs4570625-rs11178997-rs7305115 between case and control groups.

Finally, we found significant differences in the frequencies of TAGA $(P=0.005, O R=3.187,95 \%$ CI 1.376-7.382) containing rs4570625-rs11178997-rs1386494-rs7305115 between ODD and control groups (Tables 7, 8).

However, the further analysis by Haploview revealed the nominally significant finding for rs1386494 $\left(x^{2}=3.846, P=0.0499\right)$, and only one haplotypes (TAGA, $X^{2}=4.366, P=0.0367$ ) remained significant. However, the results did not remain statistically significant after 5000-fold permutation-based analysis incorporating all four SNPs or all the observed haplotypes (adjusted both $P>0.14$ ). Thus, our study was only considered as preliminary evidence of a possible association.

\section{Power analysis}

This total sample had $0.10-0.47$ power, $0.31-0.70$ power, and $0.36-0.97$ power for these four polymorphisms to detect recessive, log additive and dominant polymorphic inheritance in ODD with an odds ratio (OR) of 2 or greater (alpha $=0.05$, two tailed test).

\section{Discussion}

To our knowledge, this is the first study to find an association between ODD and the TPH-2 gene polymorphism rs1386494 or the haplotype formed by this polymorphism and other polymorphisms. While most TPH-2 association studies have used individual markers, we used polymorphism-based haplotypes and LD analysis to show that both ODD and controls shared a homogeneous LD pattern. This LD suggests that these variants segregate together in a Chinese population.

There is an extensive data consistently showing that decreased functions of the central 5-HT activity are 
Table 7 Analysis of genetic linkage disequilibrium of the four loci

\begin{tabular}{|c|c|c|c|c|c|c|}
\hline \multirow[t]{2}{*}{ SNPs } & \multicolumn{2}{|l|}{ SNP2 } & \multicolumn{2}{|l|}{ SNP3 } & \multicolumn{2}{|l|}{ SNP4 } \\
\hline & $\mathrm{D}^{\prime}$ & r2 & $\mathrm{D}^{\prime}$ & $r 2$ & $\mathrm{D}^{\prime}$ & $r 2$ \\
\hline SNP1 & 0.702 & 0.090 & 0.093 & 0.001 & 0.405 & 0.161 \\
\hline SNP2 & & & 0.653 & 0.005 & 0.001 & 0.000 \\
\hline SNP3 & & & & & 0.887 & 0.049 \\
\hline
\end{tabular}

SNP1:rs4570625; SNP2:rs11178997; SNP3:rs1386494; SNP4:rs7305115

Table 8 Haplotype analysis of TPH-2 between two groups

\begin{tabular}{|c|c|c|c|c|c|}
\hline Haplotype distribution & Case (freq) & Control (freq) & $x^{2}$ & Fisher $P$ & OR $[95 \% \mathrm{Cl}]$ \\
\hline \multicolumn{6}{|l|}{ SNP1-2 } \\
\hline G-A & $1.52(0.007)$ & $10.57(0.042)$ & 6.281 & $0.012233^{*}$ & $0.149[0.027-0.826]$ \\
\hline T-A & $40.48(0.174)$ & $24.43(0.098)$ & 6.083 & $0.013679^{*}$ & $1.951[1.140-3.341]$ \\
\hline \multicolumn{6}{|l|}{ SNP1-2-3 } \\
\hline$G-A-G$ & $1.51(0.007)$ & $10.51(0.042)$ & 6.234 & $0.012562^{*}$ & $0.149[0.027-0.831]$ \\
\hline T-A-G & $38.73(0.167)$ & $24.00(0.096)$ & 5.409 & $0.020075^{*}$ & $1.896[1.099-3.272]$ \\
\hline \multicolumn{6}{|l|}{ SNP1-2-4 } \\
\hline T-A-A & $22.24(0.096)$ & $10.60(0.042)$ & 4.981 & $0.025669^{*}$ & 2.315 [1.088-4.927] \\
\hline \multicolumn{6}{|l|}{ SNP1-2-3-4 } \\
\hline T-A-G-A & $21.77(0.094)$ & $7.79(0.031)$ & 8.031 & $0.004617^{*}$ & $3.187[1.376-7.382]$ \\
\hline
\end{tabular}

SNP1:rs4570625; SNP2:rs11178997; SNP3:rs1386494; SNP4:rs7305115* $P<0.05$

associated with uncontrolled behaviors, including impulsive behavior, aggressiveness, and substance abuse both in humans and in animal models, for example [29, 36, 37]. ТРН-2, a rate-limiting enzyme in the biosynthesis of 5-HT, is expressed mainly in brain [38]; thus, it influences the 5-HT level in brain and plays an important role in the development of mental disease [16]. Genetic variation in $T P H-2$ activity is likely to represent a critical factor in the pathogenesis of ADHD and impulsivity [16, 23]. Several studies have demonstrated that changes in the 5-HT system were critical in children with ODD [39, 40]. Moreover, the polymorphisms of TPH-2 have been shown to be associated with ADHD [16, 41, 42], obsessive-compulsive disorder [43], and bipolar affective disorder [44]. Also, TPH-2 was found to be associated with major depression [45] and pathogenesis of depression in Chinese females [46] and suicidal behavior [47, 48]. However, there has been no study reporting the relationship between TPH-2 and ODD.

This study is a case-controlled study in which the frequency of the genotypes and alleles of $T P H-2$ polymorphisms were compared between the ODD children and the control group in Chinese Han. The association between the genotypes and alleles of four candidates $T P H-2$ SNPs was investigated. Our results showed that there was a significant correlation between the frequencies of the TPH-2-rs1386494 and ODD, but not other three polymorphisms, suggesting that the locus of rs1386494 of TPH-2 was associated with ODD in Chinese Han children. The association of TPH-2-rs1386494 in this study was inconsistent with the result of Walitza et al. [23] study, in which loci of rs4570625 and rs11178997 were found to be associated with ADHD and combined ADHD and ODD, but not rs4565946. There are several reasons to explain the inconsistent results. First, the subjects in Walitza's study included both ADHD and ODD. Second, the linkage disequilibrium analysis in the core family was used in Walitza's study while the case-control association was used in several studies including our current study. Thus, the differential analysis methods and different samples may contribute to discrepant results.

Haplotypes can be more specific risk markers than single alleles, and their use reduces false-positive associations that can occur because common psychiatric disorders are likely to associate with common alleles [49]. Since the four markers analyzed were in the same haplotype block, we performed the two-four SNP sliding window haplotype analysis [50]. We found that the frequencies of the TA and GA haplotypes containing rs4570625-rs11178997, the TAG and GAG haplotypes containing rs4570625-rs11178997-rs1386494, the TAA haplotype containing rs4570625-rs11178997-rs7305115, and the TAGA haplotype containing rs4570625rs11178997-rs1386494-rs7305115 were significantly 
different between ODD and controls (all $P<0.05$ ). Further, TA, TAG, TAA and TAGA haplotypes might be the risk factors for ODD, while GA and GAG haplotypes might be protective for ODD in Chinese Han children.

Several limitations of this study should be noted. First, the number of subject children was small. The subjects of this study were 124 ODD children and 152 children in the control group. Since our sample size provided only poor statistical power, it is possible that we do see the false-positive results in the present study and our findings need to be considered cautiously. A replication study would be needed to include a large sample size. Second, although we had genotyped four polymorphisms in the present study, the coverage of genetic variation is too limited considering the total $T P H-2$ gene variants includes at least 50 polymorphisms. Therefore, it would be much better to use GWAS in larger samples to capture true positive results found in our present study. Third, the samples in our current study were only from local city in Henan province, China. Thus, the findings of this study may not be generalized for the cases of other racial or ethnic groups since the frequency of alleles can vary due to local or racial differences. Fourth, it would be better to use DSM-V as a reference rather than DSMIV. Unfortunately, we had no DSM-V when our current study was conducted. We will use DSM-V as a reference in our future investigation to remedy this shortcoming.

In summary, we report several convergent findings that implicate an effect of $T P H-2$ genotype on increased risk for ODD. We found a potential genetic association of $T P H-2$ with risk for ODD, especially the $T P H-2$ gene polymorphism rs1386494. Further haplotype analyses showed that TA, TAG, TAA and TAGA haplotypes might be the risk factors for ODD, while GA and GAG haplotypes might be protective for ODD in Chinese Han children. However, the findings in our present study remain preliminary due to the limited sample size and our low statistical power, as well as poor coverage of genetic variations in THP-2, which require replication in larger samples of ODD children from different ethnic populations.

\section{Appendix}

\begin{tabular}{|c|c|c|c|c|c|}
\hline $\begin{array}{l}\text { Sex } \\
\text { (1=boy; } \\
2=\text { girl) }\end{array}$ & Age & rs4570625 & rs11178997 & rs1386494 & rs7305115 \\
\hline 1 & 8 & GT & AT & GG & $A G$ \\
\hline 2 & 6 & $\mathrm{TT}$ & AT & GG & $A G$ \\
\hline 1 & 7 & GT & AT & GG & $A G$ \\
\hline 1 & 8 & $\mathrm{TT}$ & AT & GG & AA \\
\hline 2 & 9 & GG & $\mathrm{TT}$ & GG & GG \\
\hline
\end{tabular}

\begin{tabular}{|c|c|c|c|c|c|}
\hline $\begin{array}{l}\text { Sex } \\
\text { (1=boy; } \\
2=\text { girl) }\end{array}$ & Age & rs4570625 & rs11178997 & rs1386494 & rs7305115 \\
\hline 1 & 9 & GT & $\pi$ & GG & AA \\
\hline 2 & 7 & $\mathrm{TT}$ & $\mathrm{TT}$ & GG & AA \\
\hline 1 & 10 & $\mathrm{TT}$ & $\mathrm{TT}$ & $A G$ & AA \\
\hline 1 & 13 & $\mathrm{TT}$ & AT & GG & $A G$ \\
\hline 1 & 10 & GT & $\mathrm{TT}$ & GG & $A G$ \\
\hline 2 & 10 & GT & $\mathrm{TT}$ & GG & $A G$ \\
\hline 2 & 9 & $\mathrm{TT}$ & AT & GG & $\mathrm{AA}$ \\
\hline 2 & 7 & GT & $\mathrm{TT}$ & GG & $A G$ \\
\hline 2 & 9 & GG & $\mathrm{TT}$ & GG & GG \\
\hline 2 & 10 & GT & $\mathrm{TT}$ & $A G$ & $A G$ \\
\hline 2 & 10 & $\mathrm{TT}$ & $\mathrm{TT}$ & GG & AA \\
\hline 1 & 9 & GT & $\mathrm{TT}$ & GG & $A G$ \\
\hline 2 & 10 & $\mathrm{GT}$ & $\mathrm{TT}$ & GG & GG \\
\hline 1 & 8 & GG & $\mathrm{TT}$ & GG & GG \\
\hline 2 & 9 & GG & $\mathrm{TT}$ & GG & GG \\
\hline 1 & 10 & GG & $\mathrm{TT}$ & GG & GG \\
\hline 2 & 12 & $T T$ & AA & GG & $A G$ \\
\hline 1 & 10 & $\mathrm{TT}$ & AT & GG & AA \\
\hline 1 & 9 & GG & $\mathrm{TT}$ & GG & $A G$ \\
\hline 2 & 10 & GT & AT & GG & GG \\
\hline 2 & 10 & GT & AT & $A G$ & $\mathrm{AA}$ \\
\hline 2 & 9 & $\mathrm{TT}$ & AT & GG & $\mathrm{AA}$ \\
\hline 1 & 11 & $\mathrm{TT}$ & $\mathrm{TT}$ & GG & AA \\
\hline 1 & 14 & TT & AT & GG & $A G$ \\
\hline 2 & 14 & $\mathrm{TT}$ & $\mathrm{AA}$ & $A G$ & $A G$ \\
\hline 1 & 12 & $\mathrm{TT}$ & $\mathrm{TT}$ & GG & AA \\
\hline 2 & 14 & GT & $\mathrm{TT}$ & GG & AG \\
\hline 2 & 9 & GT & AT & GG & $A G$ \\
\hline 1 & 10 & GT & $\mathrm{TT}$ & GG & AG \\
\hline 1 & 11 & GG & $\mathrm{TT}$ & GG & $A G$ \\
\hline 1 & 12 & GG & $\mathrm{TT}$ & $A G$ & $A G$ \\
\hline 1 & 10 & TT & $\mathrm{TT}$ & GG & $A G$ \\
\hline 2 & 12 & GT & $\mathrm{TT}$ & GG & AG \\
\hline 2 & 12 & GG & $\mathrm{TT}$ & GG & AG \\
\hline 2 & 13 & GT & AT & GG & $A G$ \\
\hline 1 & 11 & GT & AT & $A G$ & AG \\
\hline 2 & 8 & GT & AT & GG & GG \\
\hline 2 & 8 & GT & $\mathrm{TT}$ & GG & $A G$ \\
\hline 1 & 8 & GT & $\mathrm{TT}$ & GG & $A G$ \\
\hline 2 & 12 & GG & $\mathrm{TT}$ & GG & GG \\
\hline 1 & 9 & GG & $\pi T$ & $A G$ & AA \\
\hline 1 & 11 & $\mathrm{GT}$ & $\mathrm{TT}$ & GG & $A G$ \\
\hline 1 & 11 & GT & $\mathrm{TT}$ & $\mathrm{GG}$ & $\mathrm{AA}$ \\
\hline 1 & 9 & TT & $\mathrm{AA}$ & GG & $A G$ \\
\hline 2 & 10 & $\mathrm{TT}$ & $\mathrm{TT}$ & GG & AA \\
\hline 1 & 7 & TT & $\mathrm{TT}$ & GG & $\mathrm{AA}$ \\
\hline 2 & 7 & GT & $\mathrm{TT}$ & GG & GG \\
\hline 1 & 11 & $\mathrm{GT}$ & AT & GG & GG \\
\hline 1 & 10 & $\mathrm{GT}$ & $\mathrm{TT}$ & GG & $A G$ \\
\hline
\end{tabular}




\begin{tabular}{|c|c|c|c|c|c|c|c|c|c|c|c|}
\hline $\begin{array}{l}\text { Sex } \\
(1=\text { boy; } \\
2=\text { girl })\end{array}$ & Age & rs4570625 & rs11178997 & rs1386494 & rs7305115 & $\begin{array}{l}\text { Sex } \\
(1=\text { boy; } \\
2=\text { girl) }\end{array}$ & Age & rs4570625 & rs11178997 & rs1386494 & rs7305115 \\
\hline 2 & 11 & GT & $\mathrm{TT}$ & GG & $A G$ & 1 & 10 & TT & $\mathrm{AA}$ & GG & $A G$ \\
\hline 1 & 10 & $\mathrm{TT}$ & $\mathrm{TT}$ & GG & GG & 2 & 10 & GT & $\mathrm{TT}$ & GG & $A G$ \\
\hline 1 & 11 & $\mathrm{TT}$ & $\mathrm{TT}$ & GG & $\mathrm{AA}$ & 1 & 8 & GG & $\mathrm{TT}$ & GG & $A G$ \\
\hline 2 & 12 & GT & $\mathrm{TT}$ & GG & AA & 1 & 9 & GT & $\mathrm{TT}$ & GG & AA \\
\hline 2 & 10 & GG & AT & GG & GG & 1 & 9 & $\mathrm{TT}$ & AT & GG & AA \\
\hline 1 & 12 & GT & AT & GG & $A G$ & 1 & 12 & GG & TT & GG & GG \\
\hline 1 & 14 & $\mathrm{TT}$ & $\mathrm{TT}$ & GG & GG & 2 & 13 & $\mathrm{TT}$ & $\mathrm{TT}$ & GG & $A G$ \\
\hline 1 & 7 & GG & $\mathrm{TT}$ & $A G$ & $\mathrm{AA}$ & 1 & 9 & $\mathrm{TT}$ & AT & GG & $A G$ \\
\hline 2 & 8 & $\mathrm{TT}$ & AA & GG & AA & 1 & 10 & GT & $\mathrm{TT}$ & GG & $\mathrm{AA}$ \\
\hline 2 & 9 & GT & $\mathrm{TT}$ & GG & $\mathrm{AA}$ & 1 & 12 & GT & AT & GG & GG \\
\hline 2 & 12 & $\pi$ & $\mathrm{TT}$ & GG & $\mathrm{AA}$ & 1 & 13 & GT & AT & GG & AA \\
\hline 1 & 9 & $\mathrm{TT}$ & AT & GG & GG & 1 & 12 & GT & AT & GG & GG \\
\hline 1 & 9 & GG & TT & GG & $A G$ & 1 & 13 & $\mathrm{TT}$ & $\mathrm{TT}$ & GG & $A G$ \\
\hline 2 & 9 & GG & TT & GG & $A G$ & 1 & 11 & & AT & GG & $\mathrm{AA}$ \\
\hline 2 & 12 & $\mathrm{TT}$ & AT & GG & GG & 1 & 12 & & AT & GG & $A G$ \\
\hline 2 & 11 & $\mathrm{TT}$ & TT & GG & $A G$ & 1 & 11 & & $\mathrm{TT}$ & GG & $A G$ \\
\hline 1 & 10 & GG & TT & GG & GG & 2 & 12 & & $\mathrm{TT}$ & GG & \\
\hline 2 & 11 & GG & TT & GG & GG & 1 & 12 & & $\mathrm{TT}$ & GG & \\
\hline 2 & 10 & GG & TT & GG & $A G$ & 1 & 11 & & $\mathrm{TT}$ & GG & \\
\hline 1 & 12 & GT & $T T$ & GG & $A G$ & 1 & 12 & & $\mathrm{TT}$ & GG & \\
\hline 2 & 11 & $\mathrm{GT}$ & TT & GG & $A G$ & 1 & 13 & & & GG & \\
\hline 2 & 11 & GT & TT & GG & $A G$ & \multicolumn{6}{|c|}{ Control group } \\
\hline 1 & 9 & GG & $\mathrm{TT}$ & GG & GG & Sex & Age & rs4570625 & rs11178997 & rs1386494 & rs7305115 \\
\hline 2 & 8 & GT & TT & GG & AG & $\begin{array}{l}\text { Sex } \\
\text { (1=boy; }\end{array}$ & Age & rs43/00<s & & & \\
\hline 1 & 12 & GT & AT & GG & AA & $2=$ girl) & & & & & \\
\hline $\begin{array}{l}1 \\
2\end{array}$ & 12 & GT & $\mathrm{TT}$ & GG & $\begin{array}{l}A A \\
A G\end{array}$ & 1 & 8 & GG & TT & GG & GG \\
\hline $\begin{array}{l}2 \\
1\end{array}$ & 7 & GT & AT & $\begin{array}{l}\text { GG } \\
\text { GG }\end{array}$ & $\begin{array}{l}A G \\
A A\end{array}$ & 2 & 7 & $\mathrm{TT}$ & $\mathrm{TT}$ & GG & $\mathrm{AA}$ \\
\hline 1 & $\begin{array}{l}14 \\
12\end{array}$ & GT & $\pi$ & GG & AA & 1 & 7 & GT & TT & $A G$ & AA \\
\hline 1 & 10 & GT & $\mathrm{TT}$ & GG & $A G$ & 2 & 9 & GT & TT & GG & $A G$ \\
\hline 1 & 12 & $\mathrm{TT}$ & TT & GG & AA & 2 & 10 & GT & TT & GG & $A G$ \\
\hline 2 & 12 & GG & $\mathrm{TT}$ & GG & $A G$ & 1 & 9 & $\mathrm{TT}$ & $\mathrm{TT}$ & GG & $\mathrm{AA}$ \\
\hline 1 & 11 & $\mathrm{TT}$ & AT & GG & AA & 2 & 8 & GT & TT & GG & $A G$ \\
\hline 1 & 13 & $\mathrm{TT}$ & $\mathrm{TT}$ & GG & $A G$ & 1 & 8 & GT & TT & $A G$ & $A G$ \\
\hline 1 & 14 & GT & $\mathrm{TT}$ & GG & $A G$ & 1 & 11 & GG & TT & GG & GG \\
\hline 2 & 8 & GG & TT & GG & $A G$ & 1 & 9 & GG & TT & GG & GG \\
\hline 1 & 8 & GT & TT & GG & $A G$ & 2 & 10 & $\mathrm{TT}$ & AT & $A G$ & $\mathrm{AA}$ \\
\hline 2 & 13 & GT & AT & GG & $A G$ & 2 & 10 & GG & TT & GG & GG \\
\hline 2 & 10 & GG & $\mathrm{TT}$ & GG & GG & 2 & 13 & GT & TT & GG & GG \\
\hline 1 & 13 & GG & $\mathrm{TT}$ & GG & GG & 1 & 11 & TT & TT & GG & $\mathrm{AA}$ \\
\hline 2 & 8 & GG & $\mathrm{TT}$ & GG & GG & 1 & 13 & $\mathrm{TT}$ & TT & GG & GG \\
\hline 2 & 9 & $\mathrm{TT}$ & $\mathrm{AA}$ & GG & GG & 2 & 7 & GT & $\mathrm{TT}$ & GG & $A G$ \\
\hline 1 & 8 & $\mathrm{GT}$ & $\mathrm{TT}$ & GG & $A G$ & 1 & 12 & GT & TT & GG & $A G$ \\
\hline 2 & 13 & $\mathrm{TT}$ & $\mathrm{TT}$ & GG & $\mathrm{AA}$ & 1 & 14 & GT & AT & GG & $\mathrm{AA}$ \\
\hline 1 & 13 & GT & AT & $\mathrm{GG}$ & $A G$ & 1 & 10 & GG & TT & $A G$ & $\mathrm{AA}$ \\
\hline 1 & 9 & $\mathrm{TT}$ & AT & GG & GG & 1 & 12 & $\mathrm{GT}$ & TT & GG & GG \\
\hline 2 & 11 & GT & TT & GG & GG & 2 & 12 & $\mathrm{TT}$ & AT & GG & $A G$ \\
\hline 1 & 13 & GG & $\mathrm{TT}$ & $\mathrm{GG}$ & GG & 2 & 11 & GG & TT & $A G$ & $A G$ \\
\hline 2 & 10 & GT & $\mathrm{TT}$ & GG & AA & 2 & 11 & GG & TT & GG & GG \\
\hline
\end{tabular}




\begin{tabular}{|c|c|c|c|c|c|c|c|c|c|c|c|}
\hline $\begin{array}{l}\text { Sex } \\
\text { (1=boy; } \\
2=\text { girl) }\end{array}$ & Age & rs4570625 & rs11178997 & rs1386494 & rs7305115 & $\begin{array}{l}\text { Sex } \\
(1=\text { boy; } \\
2=\text { girl) }\end{array}$ & Age & rs4570625 & rs11178997 & rs1386494 & rs7305115 \\
\hline 2 & 9 & GG & $\mathrm{TT}$ & GG & AA & 2 & 10 & GG & TT & GG & GG \\
\hline 2 & 11 & GT & $\mathrm{TT}$ & GG & $A G$ & 1 & 10 & GT & TT & GG & $\mathrm{AA}$ \\
\hline 2 & 12 & GT & AT & GG & $\mathrm{AA}$ & 2 & 10 & $\mathrm{TT}$ & AT & $A G$ & $\mathrm{AA}$ \\
\hline 2 & 10 & GT & $\mathrm{TT}$ & GG & AA & 2 & 11 & GT & TT & $A G$ & $\mathrm{AA}$ \\
\hline 1 & 13 & GT & $\mathrm{TT}$ & GG & AA & 2 & 10 & GT & AT & GG & $A G$ \\
\hline 1 & 9 & GG & TT & GG & GG & 2 & 10 & GT & AT & GG & $A G$ \\
\hline 1 & 9 & GT & $\mathrm{TT}$ & GG & $A G$ & 2 & 9 & GT & $\mathrm{TT}$ & GG & $A G$ \\
\hline 1 & 8 & GT & TT & GG & $\mathrm{AA}$ & 2 & 10 & GG & TT & GG & GG \\
\hline 2 & 7 & $\mathrm{TT}$ & AT & GG & $A G$ & 2 & 9 & $\mathrm{TT}$ & AT & GG & $\mathrm{AA}$ \\
\hline 2 & 10 & $\mathrm{TT}$ & $\mathrm{TT}$ & GG & $\mathrm{AA}$ & 2 & 10 & GT & AT & GG & GG \\
\hline 1 & 11 & GT & $\mathrm{TT}$ & $A G$ & AG & 2 & 10 & $\mathrm{TT}$ & TT & GG & AA \\
\hline 1 & 12 & GT & $\mathrm{TT}$ & GG & AA & 1 & 8 & GT & TT & GG & $A G$ \\
\hline 2 & 10 & GG & TT & GG & GG & 1 & 9 & $\mathrm{TT}$ & TT & $A G$ & $\mathrm{AA}$ \\
\hline 1 & 12 & $\mathrm{TT}$ & AT & GG & $A G$ & 2 & 10 & TT & TT & GG & GG \\
\hline 2 & 11 & $\mathrm{TT}$ & $\mathrm{TT}$ & GG & GG & 2 & 9 & GT & TT & GG & $A G$ \\
\hline 2 & 11 & GG & $\mathrm{TT}$ & GG & $A G$ & 1 & 10 & GT & AT & $A G$ & $A G$ \\
\hline 2 & 11 & GG & TT & GG & $A G$ & 1 & 11 & $\mathrm{TT}$ & $T T$ & $A G$ & $A G$ \\
\hline 1 & 11 & GG & $\mathrm{TT}$ & GG & GG & 1 & 12 & GT & TT & GG & $A G$ \\
\hline 2 & 12 & $\mathrm{TT}$ & $\mathrm{TT}$ & GG & GG & 2 & 13 & $\mathrm{GT}$ & TT & GG & $\mathrm{AA}$ \\
\hline 2 & 10 & GT & AT & GG & $\mathrm{AA}$ & 2 & 10 & GG & TT & GG & $A G$ \\
\hline 1 & 9 & GG & $\mathrm{TT}$ & $A G$ & AG & 2 & 11 & GT & AT & GG & GG \\
\hline 1 & 11 & GT & $\mathrm{TT}$ & GG & $A G$ & 1 & 11 & GT & TT & $A G$ & $A G$ \\
\hline 2 & 12 & GG & $\mathrm{AA}$ & GG & $A G$ & 2 & 11 & $\mathrm{TT}$ & TT & GG & $\mathrm{AA}$ \\
\hline 1 & 12 & $\mathrm{GT}$ & AT & GG & GG & 1 & 11 & GT & TT & GG & $A G$ \\
\hline 2 & 12 & GT & $\mathrm{TT}$ & GG & $A G$ & 2 & 13 & GG & $T T$ & GG & $A G$ \\
\hline 2 & 12 & GT & $\mathrm{TT}$ & GG & $A G$ & 1 & 13 & $\mathrm{TT}$ & TT & GG & $A G$ \\
\hline 2 & 9 & $\mathrm{GT}$ & $\mathrm{TT}$ & GG & GG & 2 & 13 & GT & TT & GG & $A G$ \\
\hline 1 & 9 & GT & $\mathrm{TT}$ & GG & $A G$ & 1 & 11 & $\mathrm{TT}$ & AT & GG & $A G$ \\
\hline 1 & 11 & $\mathrm{TT}$ & AA & GG & $A G$ & 2 & 10 & GG & $T T$ & GG & GG \\
\hline 2 & 14 & GT & $\mathrm{TT}$ & GG & $A G$ & 2 & 8 & GT & TT & GG & $A G$ \\
\hline 1 & 12 & GG & $\mathrm{TT}$ & GG & $A G$ & 2 & 9 & GT & TT & GG & $A G$ \\
\hline 1 & 12 & $\mathrm{GT}$ & AT & GG & $A G$ & 1 & 9 & GT & AT & GG & GG \\
\hline 1 & 12 & $\mathrm{TT}$ & TT & GG & $\mathrm{AA}$ & 1 & 10 & GT & AT & GG & GG \\
\hline 1 & 11 & GG & $\mathrm{TT}$ & GG & $A G$ & 1 & 11 & GT & TT & GG & $A G$ \\
\hline 1 & 12 & $\mathrm{GT}$ & $\mathrm{TT}$ & GG & $A G$ & 1 & 10 & GT & AT & GG & $A G$ \\
\hline 1 & 12 & GG & AT & GG & $A G$ & 1 & 11 & $\mathrm{GT}$ & AT & GG & $A G$ \\
\hline 1 & 12 & $\mathrm{GT}$ & AT & GG & GG & 2 & 10 & GG & $T T$ & GG & GG \\
\hline 1 & 12 & $\mathrm{TT}$ & TT & GG & $A G$ & 1 & 9 & GT & $\mathrm{TT}$ & $A G$ & $\mathrm{AA}$ \\
\hline 1 & 12 & $\mathrm{TT}$ & $\mathrm{TT}$ & GG & $\mathrm{AA}$ & 2 & 12 & GT & $\mathrm{AA}$ & GG & $A G$ \\
\hline 1 & 12 & GG & AT & GG & $A G$ & 2 & 12 & GT & $\mathrm{TT}$ & GG & $A G$ \\
\hline 1 & 11 & GT & AT & GG & $A G$ & 1 & 11 & GT & $T T$ & GG & $A G$ \\
\hline 1 & 10 & GG & TT & GG & GG & 1 & 7 & GT & TT & GG & $A G$ \\
\hline 2 & 11 & GT & AT & GG & GG & 1 & 10 & $\mathrm{TT}$ & TT & GG & $\mathrm{AA}$ \\
\hline 2 & 13 & GT & $\mathrm{TT}$ & GG & $\mathrm{AA}$ & 1 & 8 & GG & TT & GG & GG \\
\hline 2 & 13 & GT & $\mathrm{TT}$ & GG & $A G$ & 1 & 11 & GT & $T T$ & GG & AG \\
\hline 2 & 12 & TT & AT & GG & $A G$ & 2 & 13 & GG & TT & GG & GG \\
\hline 2 & 13 & $\mathrm{TT}$ & $\mathrm{TT}$ & GG & GG & 1 & 12 & GG & AT & GG & $\mathrm{AA}$ \\
\hline 2 & 12 & GG & TT & GG & GG & 1 & 10 & GG & TT & GG & AA \\
\hline 2 & 11 & $\mathrm{GT}$ & $\mathrm{TT}$ & AG & AA & 1 & 10 & GT & TT & GG & GG \\
\hline
\end{tabular}




\begin{tabular}{|c|c|c|c|c|c|}
\hline $\begin{array}{l}\text { Sex } \\
\text { (1=boy; } \\
2=\text { girl) }\end{array}$ & Age & rs4570625 & rs11178997 & rs1386494 & rs7305115 \\
\hline 2 & 8 & GT & TT & GG & $A G$ \\
\hline 2 & 8 & GT & AT & $A G$ & $A G$ \\
\hline 1 & 12 & GG & $\mathrm{TT}$ & GG & GG \\
\hline 1 & 12 & GG & TT & GG & GG \\
\hline 2 & 7 & $\mathrm{TT}$ & TT & GG & $A G$ \\
\hline 1 & 7 & GT & TT & $A G$ & GG \\
\hline 2 & 9 & GT & AT & GG & GG \\
\hline 2 & 10 & GT & AT & $A G$ & $A G$ \\
\hline 1 & 9 & $\mathrm{TT}$ & TT & GG & $\mathrm{AA}$ \\
\hline 2 & 10 & GT & AT & GG & GG \\
\hline 1 & 8 & GT & AT & $A G$ & GG \\
\hline 1 & 11 & GG & $\mathrm{TT}$ & GG & GG \\
\hline 1 & 9 & GG & AT & GG & $\mathrm{AA}$ \\
\hline 2 & 10 & $\mathrm{TT}$ & TT & $A G$ & AA \\
\hline 2 & 10 & GT & TT & GG & GG \\
\hline 2 & 13 & GT & TT & GG & $A G$ \\
\hline 1 & 11 & $\mathrm{TT}$ & AT & GG & $A G$ \\
\hline 1 & 11 & $\mathrm{TT}$ & TT & $A G$ & $\mathrm{AA}$ \\
\hline 2 & 7 & GT & TT & GG & \\
\hline 1 & 12 & GT & TT & GG & \\
\hline 1 & 11 & GT & AT & GG & \\
\hline 1 & 10 & GT & $\mathrm{TT}$ & GG & \\
\hline 1 & 12 & GT & AT & GG & \\
\hline 1 & 12 & $\mathrm{TT}$ & AT & GG & \\
\hline 2 & 11 & GT & AT & GG & \\
\hline 1 & 11 & GT & AT & GG & \\
\hline 2 & 9 & GG & $\mathrm{TT}$ & GG & \\
\hline 1 & 11 & GT & $\mathrm{TT}$ & GG & \\
\hline 2 & 12 & GT & TT & & \\
\hline 2 & 10 & GT & $\mathrm{TT}$ & & \\
\hline 1 & 8 & GT & $\mathrm{AA}$ & & \\
\hline
\end{tabular}

\section{Abbreviations}

ODD: oppositional defiant disorder; CD: conduct disorder; ADHD: attentiondeficit/hyperactivity disorder; TPH-2: the tryptophan hydroxylase-2 gene; $5-\mathrm{HT}$ : serotonin; IQ: intelligence quotient; PCR: polymerase chain reaction; SNP: single nucleotide polymorphisms.

\section{Authors' contributions}

C-HW and CL contributed equally for this study and are both considered as first authors, they created the design of the study and the experimental paradigm, managed the acquisition of the data, analyzed and interpreted the data, and wrote the first draft. E-ZC, G-LX and T-TL has been involved in collecting study subjects. Y-LZ did the guidance of laboratory methods in this study, Q-FN managed the acquisition of the data, analyzed and interpreted the data as an assistant, J-KW has been involved in drafting and revising the manuscript. $\mathrm{YL}$ and $\mathrm{H}-\mathrm{YN}$ are the corresponding authors and have contributed equally to this work. All authors read and approved the final manuscript.

\section{Acknowledgements}

We warmly thank the children and their parents who kindly took part in this research, as well as the schools and the teachers who agreed to collaborate in this study. We warmly thank Xiang Yang Zhang to help us modify our language errors.

\section{Competing interests}

The related authorities and the authors declare that they all have no competing interest. The authors declare that the research was conducted in the absence of any commercial, financial (and non-financial) relationships that could be construed as a potential competing interests.

\section{Consent for publication}

We have obtained the informed consent for publication from the children's parents or legal guardian.

\section{Ethics approval and consent to participate}

Research programs "Association of tryptophan hydroxylase-2 polymorphisms with oppositional defiant disorder in a Chinese Han population" have been investigated by medical ethics committee of The second affiliated hospital of Xinxiang Medical University, which result was agreement (Batch number: 20090108).

\section{Funding}

This work was supported by grants from the Medical Technology Foundation of Henan Province (142300410025 and 112102310211), Scientific Research Fund of Xinxiang Medical University (2013ZD117) and Ministry of Health research fund projects in China (20090103). Henan Provincial Department of Science and Technology Research Project (102101310400).

Received: 13 June 2016 Accepted: 4 November 2016

Published online: 21 November 2016

\section{References}

1. Riley M, Ahmed S, Locke A. Common questions about oppositional defiant disorder. Am Fam Physician. 2016;93(7):586-91.

2. Frick PJ, Nigg JT. Current issues in the diagnosis of attention deficit hyperactivity disorder, oppositional defiant disorder, and conduct disorder. Annu Rev Clin Psychol. 2012;8:77-107. doi:10.1146/ annurev-clinpsy-032511-143150.

3. Cavanagh M, Quinn D, Duncan D, Graham T, Balbuena L. Oppositional defiant disorder is better conceptualized as a disorder of emotional regulation. J Atten Disord. 2014; [Epub ahead of print]. doi:10.1177/1087054713520221.

4. Bradshaw $C P$, Schaeffer $C M$, Petras $H$, lalongo N. Predicting negative life outcomes from early aggressive-disruptive behavior trajectories: gender differences in maladaptation across life domains. J Youth Adolesc. 2010;39(8):953-66. doi:10.1007/s10964-009-9442-8.

5. Gomez R, Hafetz N, Gomez RM. Oppositional defiant disorder: prevalence based on parent and teacher ratings of Malaysian primary school children. Asian J Psychiatry. 2013;6(4):299-302. doi:10.1016/j.ajp.2013.01.008.

6. Pihlakoski L, Sourander A, Aromaa M, Rautava P. Helenius H, Sillanpää M. The continuity of psychopathology from early childhood to preadolescence: a prospective cohort study of 3 to 12-year-old children. Eur Child Adolesc Psychiatry. 2006;15(7):409-17. doi:10.1007/s00787-006-0548-1.

7. Burke JD, Loeber R, Birmaher B. Oppositional defiant disorder and conduct disorder: a review of the past 10 years, part II. J Am Acad Child Adolesc Psychiatry. 2002:41:1275-93. doi:10.1097/01.CHI.0000024839.60748.E8.

8. Tuvblad C, Zheng M, Raine A, Baker LA. A common genetic factor explains the covariation among ADHD ODD and CD symptoms in 9-10 year old boys and girls. J Abnorm Child Psychol. 2009;37(2):153-67. doi:10.1007/s10802-008-9278-9.

9. Gopin CB, Berwid O, Marks DJ, Mlodnicka A, Halperin JM. ADHD preschoolers with and without ODD: do they act differently depending on degree of task engagement/reward? J Atten Disord. 2013;17(7):608-19. doi:10.1177/1087054711432140.

10. Liu L, Cheng J, Li H, Yang L, Qian Q, Wang Y. The possible involvement of genetic variants of NET1 in the etiology of attention-deficit/hyperactivity disorder comorbid with oppositional defiant disorder. J Child Psychol Psychiatry. 2015;56(1):58-66. doi:10.1111/jcpp.12278.

11. Rowe R, Costello EJ, Angold A, Copeland WE, Maughan B. Developmental pathways in oppositional defiant disorder and conduct disorder. J Abnorm Psychol. 2010;119(4):726-38. doi:10.1037/a0020798. 
12. Coolidge FL, Thede LL, Young SE. Heritability and the comorbidity of attention deficit hyperactivity disorder with behavioral disorders and executive function deficits: a preliminary investigation. Dev Neuropsychol. 2000;17(3):273-87. doi:10.1207/s15326942dn1703_1.

13. Lahey BB, Waldman ID. Annual research review: phenotypic and causal structure of conduct disorder in the broader context of prevalent forms of psychopathology. J Child Psychol Psychiatry. 2012;53(5):536-57. doi:10.1111/j.1469-7610.2011.02509.x.

14. Faraone SV, Mick E. Molecular genetics of attention deficit hyperactivity disorder. Psychiatr Clin North Am. 2010;33(1):159-80. doi:10.1016/j. psc.2009.12.004.

15. Dittmann RW, Schacht A, Helsberg K, Schneider-Fresenius C, Lehmann M, Lehmkuhl G, Wehmeier PM. Atomoxetine versus placebo in children and adolescents with attention-deficit/hyperactivity disorder and comorbid oppositional defiant disorder: a double-blind, randomized, multicenter trial in Germany. J Child Adolesc Psychopharmacol. 2011;21(2):97-110. doi:10.1089/cap.2009.0111.

16. Park TW, Park YH, Kwon HJ, Lim MH. Association between TPH2 gene polymorphisms and attention deficit hyperactivity disorder in Korean children. Genet Test Mol Biomark. 2013;17(4):301-6. doi:10.1089/gtmb.2012.0376.

17. Marazziti D, Baroni S, Masala I, Golia F, Consoli G, Massimetti G, Picchetti M, Catena Dell'osso M, Giannaccini G. Impulsivity, gender, and the platelet serotonin transporter in healthy subjects. Neuropsychiatr Dis Treat. 2010;6:9-15.

18. Brewer JA, Potenza MN. The neurobiology and genetics of impulse control disorders: relationships to drug addictions. Biochem Pharmacol. 2008;75(1):63-75. doi:10.1016/j.bcp.2007.06.043.

19. Coccaro EF, Lee R. Cerebrospinal fluid 5-hydroxyindolacetic acid and homovanillic acid: reciprocal relationships with impulsive aggression in human subjects. J Neural Transm. 2010;117(2):241-8. doi:10.1007/s00702-009-0359-x.

20. Glick $A$. The role of serotonin in impulsive aggression, suicide, and homicide in adolescents and adults: a literature review. Int J Adolesc Med Health. 2015;27(2):143-50. doi:10.1515/ijamh-2015-5003.

21. Walther DJ, Bader M. A unique central tryptophan hydroxylase isoform. Biochem Pharmacol. 2003;66(9):1673-80. doi:10.1016/ S0006-2952(03)00556-2.

22. Sheehan K, Lowe N, Kirley A, Mullins C, Fitzgerald M, Gill M, Hawi Z. Tryptophan hydroxylase 2 (TPH2) gene variants associated with ADHD. Mol Psychiatry. 2005;10(10):944-9. doi:10.1038/sj.mp.4001698.

23. Walitza S, Renner TJ, Dempfle A, Konrad K, Wewetzer Ch, Halbach A, Herpertz-Dahlmann B, Remschmidt H, Smidt J, Linder M, Flierl L, Knölker U, Friedel S, Schäfer H, Gross C, Hebebrand J, Warnke A, Lesch KP. Transmission disequilibrium of polymorphicvariants in the tryptophan hydroxylase-2 gene in attention-deficit/hyperactivity disorder. Mol Psychiatry. 2005;10(12):1126-32. doi:10.1038/sj.mp.4001734.

24. Pereira Pde A, Romano-Silva MA, Bicalho MA, De Marco L, Correa H, de Campos SB, de Moraes EN, Torres KC, de Souza BR, de Miranda DM. Association between tryptophan hydroxylase-2 gene and late-onset depression. Am J Geriatr Psychiatry. 2011;19(9):825-9. doi:10.1097/ JGP.0b013e31820eeb21.

25. Goenjian AK, Bailey JN, Walling DP, Steinberg AM, Schmidt D, Dandekar U, Noble EP. Association of TPH1, TPH2, and 5HTTLPR with PTSD and depressive symptoms. J Affect Disord. 2012;140(3):244-52. doi:10.1016/j. jad.2012.02.015.

26. Goenjian AK, Noble EP, Steinberg AM, Walling DP, Stepanyan ST, Dandekar S, Bailey JN. Association of COMT and TPH-2 genes with DSM-5 based PTSD symptoms. J Affect Disord. 2014;172C:472-8. doi:10.1016/j. jad.2014.10.034

27. Wrzosek M, Łukaszkiewicz J, Wrzosek M, Serafin P, Jakubczyk A, Klimkiewicz A, Matsumoto H, Brower KJ, Wojnar M. Association of polymorphisms in HTR2A, HTR1A and TPH2 genes with suicide attempts in alcohol dependence: a preliminary report. Psychiatry Res. 2011;190(1):149-51. doi:10.1016/j.psychres.2011.04.027.

28. Zupanc T, Pregelj P, Paska AV. Tryptophan hydroxylase 2 (TPH-2) single nucleotide polymorphisms, suicide, and alcohol-related suicide. Psychiatr Danub. 2013;25(Suppl 2):332-6.

29. Perez-Rodriguez MM, Weinstein S, New AS, Bevilacqua L, Yuan Q, Zhou Z, Hodgkinson C, Goodman M, Koenigsberg HW, Goldman D, Siever LJ. Tryptophan-hydroxylase 2 haplotype association with borderline personality disorder and aggression in a sample of patients with personality disorders and healthy controls. J Psychiatr Res. 2010;44(15):1075-81. doi:10.1016/j.jpsychires.
30. Zhang C, Li Z, Shao Y, Xie B, Du Y, Fang Y, Yu S. Association study of tryptophan hydroxylase-2 gene in schizophrenia and its clinical features in Chinese Han population. J Mol Neurosci. 2011;43(3):406-11. doi:10.1007/ s12031-010-9458-2.

31. Xu XM, Ding $M$, Pang $H$, Wang BJ.TPH2 gene polymorphisms in the regulatory region are associated with paranoid schizophrenia in Northern Han Chinese. Genet Mol Res. 2014;13(1):1497-507. doi:10.4238/2014.March.12.1.

32. Campos SB, Miranda DM, Souza BR, Pereira PA, Neves FS, Tramontina J, Kapczinski F, Romano-Silva MA, Correa H. Association study of tryptophan hydroxylase 2 gene polymorphisms in bipolar disorder patients with panic disorder comorbidity. Psychiatr Genet. 2011;21:106-11. doi:10.1097/YPG.0b013e328341a3a8.

33. Xiao PF, Cheng L, Wan Y, Sun BL, Chen ZZ, Zhang SY, Zhang CZ, Zhou GH, Lu ZH. An improved gel-based DNA microarray method for detecting single nucleotide mismatch. Electrophoresis. 2006;27(19):3904-15. doi:10.1002/elps.200500918.

34. Xiao P, Huang H, Zhou G, Lu Z. Gel immobilization of acrylamide-modified single-stranded DNA template for pyrosequencing. Electrophoresis. 2007;28(12):1903-12. doi:10.1002/elps.200600794.

35. Gauderman WJ. Sample size requirements for association studies of gene-gene interaction. Am J Epidemiol. 2002;155(5):478-84.

36. Silva H, Iturra P, Solari A, Villarroel J, Jerez S, Jiménez M, Galleguillos F, Bustamante ML. Fluoxetine response in impulsive-aggressive behavior and serotonin transporter polymorphism in personality disorder. Psychiatr Genet. 2010;20(1):25-30. doi:10.1097/YPG.0b013e328335125d.

37. Audero E, Mlinar B, Baccini G, Skachokova ZK, Corradetti R, Gross C. Suppression of serotonin neuron firing increases aggression in mice. J Neurosci. 2013;33(20):8678-88. doi:10.1523/ JNEUROSCI.2067-12.2013.

38. Chen GL, Miller GM. Advances in tryptophan hydroxylase-2 gene expression regulation: new insights into serotonin-stress interaction and clinical implications. Am J Med Genet B Neuropsychiatr Genet. 2012;159B(2):152-71. doi:10.1002/ajmg.b.32023.

39. Martel MM, Nikolas M, Jernigan K, Friderici K, Nigg JT. Diversity in pathways to common childhood disruptive behavior disorders. J Abnorm Child Psychol. 2012;40(8):1223-36. doi:10.1007/s10802-012-9646-3.

40. Brammer WA, Lee SS. Prosociality and negative emotionality mediate the association of serotonin transporter genotype with childhood ADHD and ODD. J Clin Child Adolesc Psychol. 2013;42(6):809-19. doi:10.1080/153744 16.2013.840638.

41. Waider J, Araragi N, Gutknecht L, Lesch KP. Tryptophan hydroxylase-2 (TPH2) in disorders of cognitive control and emotion regulation: a perspective. Psychoneuroendocrinology. 2011;36(3):393-405. doi:10.1016/j. psyneuen.

42. Shim SH, Hwangbo Y, Kwon YJ, Jeong HY, Lee BH, Hwang JA, Kim YK. A case-control association study of serotonin $1 \mathrm{~A}$ receptor gene and tryptophan hydroxylase 2 gene in attention deficit hyperactivity disorder. Prog Neuropsychopharmacol Biol Psychiatry. 2010;34(6):974-9. doi:10.1016/j. pnpbp

43. Rocha FF, Alvarenga NB, Lage NV, Romano-Silva MA, Marco LA, Corrêa H. Associations between polymorphic variants of the tryptophan hydroxylase 2 gene and obsessive-compulsive disorder. Rev Bras Psiquiatr. 2011:33:176-80.

44. Carkaci-Salli N, Salli U, Tekin I, Hengst JA, Zhao MK, Gilman TL, Andrews AM, Vrana KE. Functional characterization of the S41Y (C2755A) polymorphism of tryptophan hydroxylase 2. J Neurochem. 2014;130(6):748-58. doi:10.1111/jnc.12779.

45. Gao J, Pan Z, Jiao Z, Li F, Zhao G, Wei Q, Pan F, Evangelou E. TPH2 gene polymorphisms and major depression-a meta-analysis. PLOS ONE. 2012;7(5):e36721. doi:10.1371/journal.pone.0036721.

46. Shen X, Wu Y, Qian M, Wang X, Hou Z, Liu Y, Sun J, Zhong H, Yang J, Lin M, Li L, Guan T, Shen Z, Yuan Y. Tryptophan hydroxylase 2 gene is associated with major depressive disorder in a female Chinese population. J Affect Disord. 2011;133(3):619-24. doi:10.1016/j.jad.2011.04.037.

47. Zupanc T, Pregelj P, Tomori M, Komel R, Paska AV. TPH2 polymorphisms and alcohol-related suicide. Neurosci Lett. 2011:490(1):78-81. doi:10.1016/j.neulet.2010.12.030.

48. Lazary J, Viczena V, Dome P, Chase D, Juhasz G, Bagdy G. Hopelessness, a potential endophenotpye for suicidal behavior, is influenced by TPH2 gene variants. Prog Neuropsychopharmacol Biol Psychiatry. 2012;36(1):155-60. doi:10.1016/j.pnpbp.2011.09.001. 
49. Lohmueller KE, Pearce CL, Pike M, Lander ES, Hirschhorn JN. Meta-analysis of genetic association studies supports a contribution of common variants to susceptibility to common disease. Nat Genet. 2003;33(2):177-82. doi:10.1038/ng 1071 .
50. Gabriel SB, Schaffner SF, Nguyen H, Moore JM, Roy J, Blumenstiel B, Higgins J, DeFelice M, Lochner A, Faggart M. The structure of haplotype blocks in the human genome. Science. 2002;296(5576):2225-9. doi:10.1126/science.1069424. 